data sets were obtained-22 from the right coronary artery, 10 from the left coronary artery and 24 from the circumflex artery. Overall, 44 of the 56 scans provided clear visualization of the entire artery wall for segment lengths of $24.3-63.3 \mathrm{~mm}$. In addition, the researchers demonstrated the potential of OFDI for imaging following angioplasty and stenting; OFDI following deliberate partial dissection of the arterial wall and stent placement was able to delineate the wire mesh of the stent and the remainder of the vessel. OFDI could be the key to the safe identification of vulnerable plaques in living individuals before plaque rupture.

Original article Yun SH et al. (2006) Comprehensive volumetric optical microscopy in vivo. Nat Med 12: 1429-1433

\section{Bivalirudin lessens incidence of major bleeding in acute coronary syndromes}

Patients with moderate-risk or high-risk acute coronary syndromes (ACS) benefit from an early invasive approach to treatment. Concomitant antithrombotic therapy is also recommended; however, rates of mortality and myocardial infarction remain high. Stone et al. have investigated the effects of concomitant treatment with bivalirudin, a synthetic thrombin-specific anticoagulant, and shown that it reduces the incidence of major bleeding events in patients who undergo early invasive treatment for ACS.

The study compared three antithrombotic regimens in 13,819 invasively managed patients: unfractionated heparin (or enoxaparin) plus antiplatelet therapy with a glycoprotein IIb/IIla inhibitor; bivalirudin plus a glycoprotein Ilb/IIla inhibitor; and bivalirudin monotherapy. The two regimens that used a glycoprotein Ilb/IIla inhibitor had similar 30-day rates of ischemia and major bleeding events. When compared with unfractionated heparin plus a glycoprotein Ilb/IIla inhibitor, the incidence of the composite ischemia end point with bivalirudin monotherapy was noninferior (7.3\% and $7.8 \%$, respectively; $P=0.32$ ), and monotherapy was associated with a reduced rate of major bleeding events $(5.7 \%$ vs $3.0 \%$; relative risk $0.53 ; P<0.001)$. Less than $10 \%$ of patients in the bivalirudin monotherapy arm received provisional care with glycoprotein Ilb/Illa inhibitors.

Notably, the conclusions from this study cannot be applied to noninvasive treatment strategies for ACS patients, or to other groups of patients such as those with severe renal insufficiency. Furthermore, the authors note that administering a thienopyridine, such as clopidogrel, before angiography - as occurred in $64 \%$ of study patients - might optimize the outcome of bivalirudin monotherapy.

Original article Stone GW et al. (2006) Bivalirudin for patients with acute coronary syndromes. N Engl J Med 355: 2203-2216

\section{Statins beneficial for primary prevention of CVD}

Statins improve coronary and cerebrovascular outcomes in patients with recognized cardiovascular disease (CVD). In people at risk ofbut not yet diagnosed with-CVD, the utility of these drugs as a primary preventative measure is unclear.

Thavendiranathan et al. performed a metaanalysis of seven randomized controlled trials. The studies included in this analysis had a followup period of at least 1 year, had a minimum of $80 \%$ of the trial population without CVD, and reported at least 100 CVD outcomes. The trials included a total of 42,848 patients at moderate to moderately high risk of developing CVD, of whom 21,409 received statins, and 21,439 received placebo, for a mean of 4.3 years.

Statin use reduced the relative risk of a major coronary event by $29.2 \%$ (95\% Cl 16.7-39.8\%; $P<0.001)$ and that of a major cerebrovascular event by $14.4 \%$ (95\% Cl 2.8-24.6\%; $P=0.02)$. In addition, a $33.8 \%$ decrease in the relative risk of undergoing revascularization procedures such as CABG surgery was noted in the statin group $(95 \% \mathrm{Cl} 19.6-45.5 \%$; $P<0.001)$. There were, however, no significant differences between the placebo and statin groups with regard to mortality, either overall or as a result of coronary heart disease. The authors suggest that routine statin therapy could be a cost-effective means of primary prevention of coronary heart disease, but only for those at high risk of major coronary events.

Original article Thavendiranathan P et al. (2006) Primary prevention of cardiovascular diseases with statin therapy. Arch Intern Med 166: 2307-2313 